\title{
Haemophagocytic lymphohistiocytosis in patients treated with immune checkpoint inhibitors: analysis of WHO global database of individual case safety reports
}

\author{
Roberta Noseda ${ }^{1 *}$ D, Raffaela Bertoli ${ }^{1}$, Laura Müller ${ }^{1}$ and Alessandro Ceschi ${ }^{1,2}$
}

\begin{abstract}
Background: Immune checkpoint inhibitor (ICI) use in clinical practice has unravelled a spectrum of immunerelated adverse events (irAEs) due to immune system hyper-activation. ICl-related haemophagocytic lymphohistiocytosis $(H L H)$ has been recently outlined in single case reports, raising a concern about the need of increasing our knowledge on this rare yet life threatening ICI haematological toxicity.

Methods: To determine ICl-related HLH clinical, haematological, and coagulation features, its timing and outcome, concurrent irAEs and concomitant infections, we performed a retrospective observational cross-sectional study and queried VigiBase, the WHO global database of suspected adverse drug reactions (ADRs), on September 30th, 2018. We retrieved the individual case safety reports reporting $\mathrm{HLH}$ in association with ipilimumab, nivolumab, pembrolizumab, atezolizumab, avelumab or durvalumab, gathered in the database starting from the ICls' approval dates by the US Food and Drug Administration. The main outcome measures were co-suspected drugs, concurrent irAEs, HLH clinical, haematological and coagulation features, concomitant infections, HLH median time to onset and outcome.

Results: Among 49'883 ICl-related ADRs collated in VigiBase as of September 30th, 2018, HLH was reported in 38 cases of which 34 (90\%) mentioned ICls as the solely suspected drugs. ICl-related HLH showed clinical, haematological and coagulation features similar to those of HLH with different etiology. Concurrent irAEs occurred in 5 (13\%) patients and 6 (16\%) reported concomitant viral infections. 31 (82\%) cases defined ICI-related HLH outcome, which resolved in 19 (61\%) cases. HLH developed a median of 6.7 weeks after initiation of ICI treatment (IQR 2.9-15.4, $n=18,47 \%)$.

Conclusions: By evaluating the largest cohort of ICl-related HLH cases, we observed that ICl-related HLH arises with a delayed timing with respect to initiation of $\mathrm{ICl}$ treatment, and usually presents without other irAEs and concomitant infections. Keeping in mind these findings, clinicians should consider ICls' involvement in the onset of $\mathrm{HLH}$ whenever they diagnose a disease of this group of syndromes in cancer patients treated with $\mathrm{ICls}$.
\end{abstract}

Keywords: Immune checkpoint inhibitor, Haemophagocytic lymphohistiocytosis, VigiBase

\footnotetext{
* Correspondence: roberta.noseda@eoc.ch

'Division of Clinical Pharmacology and Toxicology, Institute of

Pharmacological Sciences of Southern Switzerland, Ente Ospedaliero

Cantonale, Via Tesserete 46, 6903 Lugano, Switzerland

Full list of author information is available at the end of the article
}

(c) The Author(s). 2019 Open Access This article is distributed under the terms of the Creative Commons Attribution 4.0 International License (http://creativecommons.org/licenses/by/4.0/), which permits unrestricted use, distribution, and reproduction in any medium, provided you give appropriate credit to the original author(s) and the source, provide a link to the Creative Commons license, and indicate if changes were made. The Creative Commons Public Domain Dedication waiver (http://creativecommons.org/publicdomain/zero/1.0/) applies to the data made available in this article, unless otherwise stated. 


\section{Background}

Haemophagocytic lymphohistiocytosis (HLH) is a rare life threatening group of syndromes characterised by hyper-activation of the immune system, which can lead to progressive organ damage and death [1]. According to the etiology, HLH divides into primary (genetic) and secondary (acquired). The latter is usually triggered by more than one external causative factors (e.g. infections and exposure to drugs with immunomodulatory effects) and underlying autoimmune diseases as well as neoplasms can increase the risk of HLH [2].

Nowadays, cancer immunotherapy with immune checkpoint inhibitors (ICIs) is progressively becoming a cornerstone in the treatment of a number of cancer types. However, by removing the normal inhibitory control that negatively regulates $\mathrm{T}$-cell function, ICIs may result in T-cells hyper-activation and immune-related adverse events (irAEs) [3]. Therefore, ICI toxicity appears to partially overlap the dysregulated immune activation characterising HLH. In literature, information on $\mathrm{HLH}$ during treatment with ICIs is limited to a handful of case reports showing a broad range of symptoms, different therapeutic interventions and different outcomes [4-12]. We used VigiBase, the World Health Organization (WHO) global database of individual case safety reports [13] to describe the largest-to-date cohort of ICI-related HLH cases reported in clinical practice. Increasing knowledge on ICI-mediated HLH clinical presentation, timing, and outcome might facilitate the recognition of this multifaceted haematological toxicity, which could be erroneously attributed uniquely to the underlying cancer or other systemic inflammatory processes.

\section{Methods}

VigiBase (http://www.vigiaccess.org/) is the largest pharmacovigilance database in the world that gathers spontaneously reported individual case safety reports of suspected adverse drug reactions (ADRs). The safety reports originate from over 130 member countries participating to the WHO Programme for International Drug Monitoring. Different types of reporters (e.g. physicians, pharmacists, other health care professionals, and patients) can contribute to generate safety reports, whereas a small proportion of reports derives from clinical studies. The scope of VigiBase is to identify novel ADRs and to gain knowledge on specific features of ADRs (e.g. spectrum, time to onset, and outcome) [13]. In VigiBase, reports are recorded in a structured form and include general administrative information (country of origin, reporting date, case seriousness), patient characteristics (age, sex), drugs (indication, start and end dates), and reactions (reported and coded term, onset date, outcome) details. The Medical Dictionary for Regulatory Activities (MedDRA - version 21.0 at the time of the study) allows for reactions' coding into hierarchal groups. For this retrospective observational cross-sectional study, we selected cases reporting the MedDRA preferred term "histiocytosis haematophagic", which comprised histiocytosis haemophagocytic, haemophagocytic lymphohistiocytosis, haemophagocytic syndrome, and macrophage activation syndrome. For clarity, throughout the manuscript, we used the term HLH to refer to this group of syndromes. We queried VigiBase on September 30th, 2018, for HLH reports associated with ipilimumab, nivolumab, pembrolizumab, atezolizumab, avelumab, and durvalumab, gathered in the database starting from the approval date of each substance by the United States Food and Drug Administration. We assessed co-suspected drugs, concurrent irAEs, HLH clinical, haematological and coagulation features, concomitant infections, HLH median time to onset and outcome.

To get further insights into patient comorbidities, we searched in PubMed for case reports of ICI-related HLH (applying the same inclusion criteria as in the VigiBase query), and subsequently selected those matching with the safety reports we retrieved from VigiBase. We used frequency and percentage to summarize categorical variables, and median and interquartile range (IQR) for continuous variables. Analyses were carried out by Microsoft Excel (2010, Microsoft Corporation, Washington, USA).

\section{Results}

Among 49'883 ICI-related ADRs collated in VigiBase as of September 30th, 2018, HLH was reported in 38 cases. The highest reporting rate of ICI-related HLH occurred in France $(0.4 \%)$, whereas the lowest in the United States of America (0.03\%, Table 1). Table 2 resumes the baseline characteristics of the identified reports. 29 (76\%) involved males and median patient age was 63 years (IQR 45-72 years). Melanoma was the most common cancer type $(21,55 \%)$, followed by lung cancer $(5,13 \%$ non-small cell lung cancer, $n=4$, adenocarcinoma of lung, $n=1$ ). ICIs were the solely suspected drugs in 34

Table 1 Geographical pattern of immune checkpoint inhibitorrelated haemophagocytic lymphohistiocytosis reporting rate

\begin{tabular}{llll}
\hline $\begin{array}{l}\text { Country of } \\
\text { primary source }\end{array}$ & $\begin{array}{l}\text { Total number of } \\
\text { ICl-related } \\
\text { safety reports }\end{array}$ & $\begin{array}{l}\text { Number of ICl-related } \\
\text { HLH safety reports }\end{array}$ & $\begin{array}{l}\text { ICl-related } \mathrm{HLH} \\
\text { reporting } \\
\text { rate (\%) }\end{array}$ \\
\hline France & 3526 & 14 & 0.4 \\
Japan & 6421 & 11 & 0.2 \\
Germany & 1901 & 3 & 0.2 \\
Switzerland & 830 & 1 & 0.1 \\
Canada & 1279 & 1 & 0.08 \\
US of America & $24 ' 998$ & 8 & 0.03 \\
\hline
\end{tabular}

Abbreviations: $\mathrm{ICl}$ immune checkpoint inhibitor, $\mathrm{HLH}$ haemophagocytic lymphohistiocytosis 
Table 2 Baseline characteristics of the individual case safety reports concerning immune checkpoint inhibitor-related haemophagocytic lymphohistiocytosis

\begin{tabular}{|c|c|}
\hline Characteristic & $\begin{array}{l}\text { Patients No. (\%) } \\
(n=38)\end{array}$ \\
\hline \multicolumn{2}{|l|}{ Age } \\
\hline Reported & $33(87)$ \\
\hline Median $[\mathrm{IQR}]$, years & $63[45-72]$ \\
\hline Not reported & $5(13)$ \\
\hline \multicolumn{2}{|l|}{ Sex } \\
\hline Male & $29(76)$ \\
\hline Female & $9(24)$ \\
\hline \multicolumn{2}{|l|}{ Cancer type } \\
\hline Melanoma & $21(55)$ \\
\hline Lung cancer & $5(13)$ \\
\hline Bladder cancer & $3(8)$ \\
\hline Renal cell carcinoma & $2(5)$ \\
\hline Hodgkin disease & $1(3)$ \\
\hline Transitional cell carcinoma & $1(3)$ \\
\hline Adenocarcinoma gastric & $1(3)$ \\
\hline Thymoma & $1(3)$ \\
\hline T cell lymphoblastic leukemia acute & $1(3)$ \\
\hline Not reported & $2(5)$ \\
\hline \multicolumn{2}{|l|}{ Co-suspected drugs } \\
\hline Reported & $4(10)$ \\
\hline Antineoplastic agents & $2(5)$ \\
\hline Antibacterial agents & $2(5)$ \\
\hline Not reported & $34(90)$ \\
\hline \multicolumn{2}{|l|}{ Drugs } \\
\hline Anti-CTLA-4 (ipilimumab) monotherapy & $7(18)$ \\
\hline \multicolumn{2}{|l|}{ Anti-PD-1 monotherapy } \\
\hline nivolumab & $14(37)$ \\
\hline pembrolizumab & $7(18)$ \\
\hline \multicolumn{2}{|l|}{ Anti-PD-L1 monotherapy } \\
\hline Atezolizumab & $1(3)$ \\
\hline ipilimumab and nivolumab combination therapy & $5(13)$ \\
\hline nivolumab and ipilimumab sequential therapy & $3(8)$ \\
\hline pembrolizumab and ipilimumab sequential therapy & $1(3)$ \\
\hline \multicolumn{2}{|l|}{ Reporting } \\
\hline 2014 & $1(3)$ \\
\hline 2015 & $3(8)$ \\
\hline 2016 & $6(16)$ \\
\hline 2017 & $10(26)$ \\
\hline 2018 & $18(47)$ \\
\hline
\end{tabular}

Abbreviations: IQR interquartile range, CTLA-4 Cytotoxic T-Lymphocyte Antigen 4, $P D-1$ Programmed cell Death protein 1, $P D-L 1$ Programmed cell Death-Ligand 1
(90\%) cases, whereas 4 (10\%) patients reported as additional suspected drugs other antineoplastic agents $(n=2)$ and antibacterial agents $(n=2)$. Out of 38 cases of ICI-associated HLH, 22 (58\%) received an anti-Programmed-Death-1/Programmed-Death-Ligand1 agent (PD-1/PD-L1). Regarding treatment duration, 4 (11\%) patients received a single administration, 19 (50\%) had a prolonged treatment (median duration 9.9 weeks, IQR 5.9-25.9 weeks), and in 15 (39\%) patients treatment duration could not be defined. ICI-mediated HLH developed a median of 6.7 weeks after initiation of ICI treatment (IQR 2.9-15.4 weeks, $n=18,47 \%$ ), and HLH reporting in association with ICIs increased over time (18 cases, $47 \%$, in 2018 , at the time of writing).

All cases of ICI-related HLH were evaluated by reporters as serious for causing or prolonging hospitalization $(16,42 \%)$, for determining life threatening conditions $(7,18 \%)$, or because related to death $(10$, $26 \%$ ). Five (13\%) cases did not specify the seriousness criteria. Among the ten fatal cases, $4(40 \%)$ mentioned HLH as the unique cause of death. In three (30\%) patients, HLH contributed to death along with malignant neoplasm progression, multi-organ failure, and brain haemorrhage at the cerebral metastasis site, respectively. In the remaining $3(30 \%)$ cases reporting death, HLH probably did not contribute to death which occurred either for sepsis $(n=1)$ or malignant neoplasm progression $(n=2)$. Besides fatal cases, HLH was not resolved at the time of reporting in $5(16 \%)$ patients whereas recovered in $19(61 \%)$ patients (out of 31 cases defining HLH outcome).

As shown in Table 3, concurrent irAEs occurred in 5 (13\%) patients and HLH clinical, haematological and coagulation features were mentioned in 15 (\%) cases. Concomitant viral infections were reported in $6(16 \%)$ cases (Herpes zoster, $n=1$; Epstein-Barr virus, $n=5$ ).

When we searched the literature for case reports of ICI-associated HLH, we identified six cases matching with individual case safety reports retrieved from VigiBase. However, none of these case reports allowed gaining information on patients' comorbidities.

\section{Discussion}

We reported the largest-to-date analysis of ICI-associated HLH cases collated in the WHO global database of suspected ADRs. By blocking the signalling pathway that negatively regulates $\mathrm{T}$ cell activation, ICIs enhance the immune response against cancer cells and, aberrantly, against self-antigens triggering immune-related adverse events [3]. Hyper-activation of lymphocytes, natural killer (NK) cells and histiocytes are distinguishing features of HLH [1]. To date, a causative role for activated T cells in HLH development has been hypothesized in cancer 
Table 3 Haemophagocytic lymphohistiocytosis features and immune-related adverse events reported in patients treated with immune checkpoint inhibitors

\begin{tabular}{|c|c|}
\hline Clinical features of $\mathrm{HLH}$ & $\begin{array}{l}\text { Patients No. }(\%)^{a} \\
(\mathrm{n}=38)\end{array}$ \\
\hline Pyrexia & $2(5)$ \\
\hline \multicolumn{2}{|l|}{ Pulmonary involvement } \\
\hline Cough & $1(3)$ \\
\hline \multicolumn{2}{|l|}{ Neurological involvement } \\
\hline Encephalopathy & $1(3)$ \\
\hline Headache & $1(3)$ \\
\hline \multicolumn{2}{|l|}{ Psychiatric changes } \\
\hline Delirium & $1(3)$ \\
\hline \multicolumn{2}{|l|}{ Cutaneous involvement } \\
\hline Generalised erythema & $1(3)$ \\
\hline Drug eruption & $1(3)$ \\
\hline \multicolumn{2}{|l|}{ Gastrointestinal involvement } \\
\hline Enterocolitis & $1(3)$ \\
\hline Diarrhoea & $2(5)$ \\
\hline \multicolumn{2}{|l|}{ Renal involvement } \\
\hline Renal failure & $1(3)$ \\
\hline Renal tubular necrosis & $1(3)$ \\
\hline \multicolumn{2}{|l|}{ Haematological and coagulation features of $\mathrm{HLH}$} \\
\hline Anaemia & $1(3)$ \\
\hline Thrombocytopenia & $2(5)$ \\
\hline Leukopenia/ White blood cell count decreased & $2(5)$ \\
\hline Neutrophil count decreased & $1(3)$ \\
\hline Disseminated intravascular coagulation & $3(8)$ \\
\hline International normalised ratio abnormal & $1(3)$ \\
\hline Pancytopenia/ Bone marrow failure & $2(5)$ \\
\hline \multicolumn{2}{|l|}{ Concurrent irAEs } \\
\hline Autoimmune hepatitis & $2(5)$ \\
\hline Interstitial lung disease & $2(5)$ \\
\hline Myositis & $1(3)$ \\
\hline Thyroiditis & $1(3)$ \\
\hline Cardiac $^{b}$ & $1(3)$ \\
\hline
\end{tabular}

Abbreviations: HLH haemophagocytic lymphohistiocytosis, irAEs immunerelated adverse events

${ }^{a}$ Some patients reported more than one adverse drug reaction besides HLH

${ }^{\mathrm{b}}$ Atrial fibrillation and left ventricular failure

patients as an additive effect to the excessive cytokine secretion induced by cancer cells $[2,14]$.

Remarkably, VigiBase does not provide data on the total number of patients treated with ICIs in clinical practice; therefore, the absolute incidence of HLH from ICI usage cannot be evaluated with such a source of data. Conversely, by gathering spontaneous safety reports from more than 130 countries, VigiBase allows for the assessment of the proportion of safety reports for a specific drug toxicity (i.e. ICI-related HLH) out of the total number of safety reports present in the database for the drug of interest (i.e. all ICI-related ADRs). In light of this evidence, we could observe that HLH was included in less than $0.1 \%$ of all safety reports associated with ICIs overall, thus confirming that it is a rare haematological toxicity upon treatment with ICIs.

We observed geographical variability of ICI-related HLH reporting rate across countries of primary source; in particular, we found that France, Germany and Japan had the highest ICI-related HLH reporting rates whereas the US had the lowest. This might suggest a genetic predisposition towards ICI haematological toxicity and HLH development, similarly to previous observations relating specific genetic backgrounds to different patients' predisposition towards HLH triggering agents [2]. Moreover, we found that ICI-related HLH safety reports mostly involved melanoma patients, probably due to the earlier approval of ipilimumab and anti-PD-1 agents for melanoma, which, consistently, were the substances most commonly reported as suspected by the majority of ICI-related HLH safety reports.

The reporting of HLH as ICI-mediated toxicity is increasing over years. Although this likely depends on the progressively increasing use of ICIs across different cancer types, our study strengthens awareness of a novel and relevant pharmacological trigger for $\mathrm{HLH}$, in addition to neoplasms and infections as well-known HLH predisposing factors [2]. Indeed, the majority of study cases mentioned neither underlying malignancies nor concomitant infections as HLH contributory causes besides ICIs. However, these findings must be adequately weighted when interpreted as, relying on spontaneous reporting, individual case safety reports are rarely sufficient to confirm that a specific drug caused the adverse event [13], and do not rule out the existence of other contributing causes of the reported ADR, e.g. the disease being treated, a new disease, other ADRs of the same drug, or other suspected drugs. Remarkably, the latter were mostly absent from the cases we analysed, suggesting ICIs pivotal role as contributing pharmacological trigger in the genesis of HLH.

Although the quality of the large amount of data stored in VigiBase is variable in that the reported information derives from different sources, with different levels of details and sometimes partial or missing data, VigiBase represents a unique source of information, allowing for the detection of the rarer and less frequently reported ADRs [13]. By exploiting this database, we managed to identify 38 cases of ICI-related HLH, far beyond the amount of cases published to date in the literature [4-12].

Since patient comorbidities are sparsely reported in VigiBase, with co-suspected and concomitant drugs 
providing limited insights, to try to overcome this limitation, we also reviewed published single case reports [412] that matched with the safety reports retrieved from VigiBase. Unfortunately, the information retrieved from these case reports was insufficient to identify predisposing conditions shared among patients developing HLH on ICI treatment.

HLH as adverse effect of drugs has been rarely reported in literature and mainly described in association with the systemic inflammatory response induced by drug reaction with eosinophilia and systemic symptoms (DRESS) syndrome [15], or as a consequence of an infection secondary to immunosuppressant agents [2]. More recently, antiepileptic drugs were associated with HLH onset, because of their immune-modulating action [16, 17]. Noteworthy, anticonvulsant drug-related HLH developed 2-3 weeks after treatment initiation, a time to onset relatively shorter if compared with the delayed time to onset that we observed with ICIs.

Confirming that HLH is a life threatening condition, reporters evaluated all ICI-related HLH cases as serious, although HLH resolved in the majority of cases, in agreement with the notion that this group of syndromes generally responds to systemic corticosteroids [2].

\section{Conclusions}

In clinical practice, ICI-related HLH arises with a delayed timing with respect to initiation of ICI treatment, presents clinical, haematological and coagulation features similar to those of HLH with different etiology, rarely is associated with other irAEs and concomitant viral infections, and resolved in the majority of cases. Keeping in mind these findings, clinicians should consider ICIs' involvement in the onset of HLH whenever they diagnose a disease of this group of syndromes in cancer patients treated with ICIs.

\section{Abbreviations \\ ADR: Adverse Drug Reaction; DRESS: Drug Reaction with Eosinophilia and Systemic Symptoms; HLH: Haemophagocytic lymphohistiocytosis; ICl: Immune Checkpoint Inhibitor; IQR: Interquartile Range; irAE: Immune- related Adverse Event; MedDRA: Medical Dictionary for Regulatory Activities; WHO: World Health Organization}

\section{Acknowledgements}

Not applicable.

Funding

No funding.

\section{Availability of data and materials}

The dataset generated and analysed during the current study is available at http://www.vigiaccess.org/, by applying the inclusion criteria defined in the Methods.

\section{Authors' contributions}

$\mathrm{RN}$ analysed and interpreted the patient data regarding haemophagocytic lymphohistiocytosis adverse drug reactions associated with immune checkpoint inhibitor treatments. RN wrote the manuscript. AC, RB and LM edited the manuscript. All authors read and approved the final manuscript.

\section{Ethics approval and consent to participate}

Ethical approval was not required according to the Swiss Human Research Act (no intervention with human subjects or access to identifiable information).

\section{Consent for publication}

Not applicable.

\section{Competing interests}

The authors declare that they have no competing interests.

Study results and conclusions are those of the authors and do not necessarily represent the opinion of the $\mathrm{WHO}$.

\section{Publisher's Note}

Springer Nature remains neutral with regard to jurisdictional claims in published maps and institutional affiliations.

\section{Author details}

${ }^{1}$ Division of Clinical Pharmacology and Toxicology, Institute of Pharmacological Sciences of Southern Switzerland, Ente Ospedaliero Cantonale, Via Tesserete 46, 6903 Lugano, Switzerland. ²Department of Clinical Pharmacology and Toxicology, University Hospital Zurich, Zurich, Switzerland.

Received: 13 December 2018 Accepted: 22 April 2019

Published online: 02 May 2019

\section{References}

1. Henter JI, Elinder G. Ost a, and the the FHL study Group of the Histiocyte Society. Diagnostic guidelines for hemophagocytic lymphohistiocytosis. Semin Oncol. 1991;(1):29-33.

2. Ramos-Casals M, Brito-Zerón P, López-Guillermo A, Khamashta MA, Bosch X. Adult haemophagocytic syndrome. Lancet. 2014;383(9927):1503-16.

3. Postow MA, Sidlow R, Hellmann MD. Immune-related adverse events associated with immune checkpoint blockade. N Engl J Med. 2018;378:15868.

4. Daver N, Kantarjian H. Malignancy-associated haemophagocytic lymphohistiocytosis in adults. Lancet Oncol. 2017;(2):169-71.

5. Shah D, Shrestha R, Ramlal R, Hatton J, Saeed H. Pembrolizumab associated hemophagocytic lymphohistiocytosis. Ann Oncol. 2017;28(6):1403.

6. Takeshita M, Anai S, Mishima S, Inoue K. Coincidence of immunotherapyassociated hemophagocytic syndrome and rapid tumor regression. Ann Oncol. 2017;28(1):186-9.

7. Sasaki K, Uehara J, linuma S, Doi H, Honma M, Toki Y, et al. Hemophagocytic lymphohistiocytosis associated with dabrafenib and trametinib combination therapy following pembrolizumab administration for advanced melanoma. Ann Oncol. 2018;29(7):1602-3.

8. Satzger I, Ivanyi $P$, Länger $F$, Kreipe $H H$, Schaper-Gerhardt $K$, Beutel $G$, et al. Treatment-related hemophagocytic lymphohistiocytosis secondary to checkpoint inhibition with nivolumab plus ipilimumab. Eur J Cancer. 2018; 93:150-3.

9. Malissen N, Lacotte J, Du-Thanh A, Gaudy-Marqueste C, Guillot B, Grob JJ. Macrophage activation syndrome: a new complication of checkpoint inhibitors. Eur J Cancer. 2017;77:88-9.

10. Sadaat M, Jang S. Hemophagocytic lymphohistiocytosis with immunotherapy: brief review and case report. J Immunother Cancer. 2018; 6(1):49.

11. Michot JM, Pruvost R, Mateus C, Champiat S, Voisin AL, Marabelle A, et al. Fever reaction and haemophagocytic syndrome induced by immune checkpoint inhibitors. Ann Oncol. 2018;29(2):518-20.

12. Hantel A, Gabster B, Chenj JX, Golomb H, Gajewski TF. Severe hemophagocytic lymphohistiocytosis in a melanoma patient treated with ipilimumab + nivolumab. J Immunother Cancer. 2018;6(1):73.

13. Lindquist M. VigiBase, the WHO global ICSR database system: basic facts. Drug Inf J. 2008;42(5):409-19.

14. Ammann S, Lehmberg K, Zur Stadt U, Janka G, Rensing-Ehl A, Klemann C, et al. Primary and secondary hemophagocytic lymphohistiocytosis have 
different patterns of T-cell activation, differentiation and repertoire. Eur J Immunol. 2017;47:364-73.

15. Lambotte O, Costedoat-Chalumeau N, Amoura Z, Piette JC, Cacoub P. Druginduced hemophagocytosis. Am J Med. 2002;112:592-3.

16. Yang YC, Jou ST, Chang YH, Liang JS, Lee WT. Hemophagocytic syndrome associated with antiepileptic drug. Pediatr Neurol. 2004;30:358-60.

17. Lakhoua G, Aouinti I, Sahnoun R, Kastalli S, Daghfous R, Zaïem A. A hemophagocytosis syndrome attributed to phenobarbital. Presse Med. 2016;45(3):379-81.

Ready to submit your research? Choose BMC and benefit from:

- fast, convenient online submission

- thorough peer review by experienced researchers in your field

- rapid publication on acceptance

- support for research data, including large and complex data types

- gold Open Access which fosters wider collaboration and increased citations

- maximum visibility for your research: over $100 \mathrm{M}$ website views per year

At $\mathrm{BMC}$, research is always in progress. 